\title{
Logoclonia might be a Characteristic of Logopenic Variant Primary Progressive Aphasia at an Advanced Stage: Potential Mechanisms Underlying Logoclonia
}

\author{
Yoshitaka Nakagawa ${ }^{\mathrm{a}, *}$, Michitaka Funayama ${ }^{\mathrm{b}}$ and Masahiro Kato ${ }^{\mathrm{c}}$ \\ ${ }^{a}$ Department of Rehabilitation, Edogawa Hospital, Tokyo, Japan \\ ${ }^{\mathrm{b}}$ Department of Neuropsychiatry, Ashikaga Red Cross Hospital, Tochigi, Japan \\ ${ }^{\mathrm{c}}$ Department of Neurology, Edogawa Hospital, Tokyo, Japan
}

Accepted 9 May 2019

\begin{abstract}
Logoclonia, which is the meaningless repetition of a syllable, particularly an end syllable of a word, has been described in patients with dementia for a century. The mechanisms behind logoclonia, however, have yet to be clarified. Among 914 patients with aphasia, five patients presented with logoclonia, all of whom were categorized as having logopenic variant PPA (lvPPA) during the initial stage of their illness and met the clinical criteria for diagnosis of probable Alzheimer's disease. Cognitively, they were all severely impaired when they presented with logoclonia. During the progression from lvPPA to logoclonia in these patients, their naming abilities and phonological output function deteriorated despite their retained speech fluency. Logoclonia might be a characteristic sign of advanced-stage lvPPA. Although logoclonia might be associated with perseveration, deterioration in naming abilities and phonological output function along with retained speech fluency might form the basis for the development of logoclonia.
\end{abstract}

Keywords: Alzheimer's disease, logoclonia, logopenic variant primary progressive aphasia, naming abilities, phonological output

\section{INTRODUCTION}

Logoclonia refers to meaningless repetition of a syllable, especially an end syllable of a word. It was first reported among individuals with dementia by Kraepelin 1910 [1], who described it as a severe linguistic dysfunction observed in the advanced stages of early-onset Alzheimer's disease. Logoclonia has been described by several neurologists since then and has often been linked to Alzheimer's disease

\footnotetext{
*Correspondence to: Yoshitaka Nakagawa, Department of Rehabilitation, Edogawa Hospital, 2-24-18, Higashikoiwa, Edogawa-ku, Tokyo 133-0052, Japan. Tel.: +81 33673 1221; Fax: +81 33673 1229; E-mail: rehabilitation@edogawa.or.jp.
}

[2-6], particularly advanced-stage disease [6]. Logoclonia has also been associated with basal ganglia function or degenerative extrapyramidal syndrome [7-9]. In that case, however, patients with logoclonia also present with other recurring utterances such as palilalia [7-9]. Palilalia and stuttering have disorders that are similar to those of logoclonia and have been associated with Parkinson's disease and progressive supranuclear palsy [10-16]. Palilalia, however, refers to the repetition of an entire word, but not a syllable. Stuttering is the involuntary disruption or blocking of speech with sound repetition, especially the initial consonants of a word, and, unlike logoclonia, stuttering is accompanied by tense and effortful utterance. Whereas patients with logoclonia have aphasia 
[1-6], those who present with palilalia or stuttering do not necessarily suffer from aphasia and instead are associated with degenerative extrapyramidal syndrome [10-16]. Considering these differences, in this manuscript, logoclonia was defined as the repetition of a middle or end syllable of a word to clearly distinguish it from the other two signs.

Studies of the mechanisms behind logoclonia, none of which have been comprehensive or systematic, have yet to clarify its origin(s). Some researchers associate logoclonia with perseveration [1]. If this is true, logoclonia might be found more frequently in patients with frontotemporal lobar degeneration than in those with Alzheimer's disease, because perseveration is one of the characteristics of frontal lobe dysfunction [17] and behavior variant frontotemporal dementia [18]. Thus, we chose to explore the potential mechanisms underlying logoclonia. We describe here the development of logoclonia in five individuals in detail and suggest some hypotheses about logoclonia.

\section{MATERIALS AND METHODS}

\section{Subjects}

Ethical aspects of this study and study protocol was approved by the Edogawa Hospital Human Research Ethics Committee. Patients with aphasia were recruited from the rehabilitation department at Edogawa Hospital and the Cognitive Function Clinic at Ashikaga Red Cross Hospital between January 2008 and December 2017. Both facilities are specialized in the treatment and rehabilitation of patients with cognitive dysfunction, i.e., acquired brain injury and degenerative diseases, in particular, patients with aphasia. In these facilities, the free conversation of all aphasic patients at the beginning of each therapy session is transcribed, which allows the identification of signs of logoclonia, i.e., the repetition of a middle or end syllable of a word. All patients were diagnosed by both neurologists and neuropsychiatrists, each of whom had more than 10 years of experience in neuropsychology and clinical practice for degenerative disorders at the time of the study. Among 914 patients with aphasia (762 at Edogawa hospital and 152 at Ashikaga Red Cross Hospital), five patients developed logoclonia over time, all of whom were categorized as having logopenic variant PPA (lvPPA) during the initial stage of their illness. Among the initial 914 patients with aphasia, 47 cases (29 at Edogawa and 18 at Ashikaga), who were all Japanese, met both the clinical and imaging-supported diag- nostic criteria for primary progressive aphasia (PPA) [19]. Based on the clinical observation for more than 1 year and brain images, they were categorized into three variants of PPA by using Gorno-Tempini's classification criteria $[19,20]$ : 16 patients were categorized as having non-fluent agrammatic variant PPA, 16 as having logopenic variant PPA, and 15 as having semantic variant PPA. Regarding diseaselevel classifications such as progressive supranuclear palsy, corticobasal degeneration, and motor neuron diseases, 5 of the 16 patients with non-fluent agrammatic variant PPA were diagnosed as having possible progressive supranuclear palsy, 2 as having motor neuron disease, and 1 as having possible corticobasal degeneration, whereas only 1 of the 15 patients with semantic variant PPA and 1 of the 16 patients with logopenic variant PPA had motor neuron disease and possible corticobasal degeneration, respectively. The observation period from the onset of PPA to the last assessment was not statistically different among the three variants (ANOVA, $p=0.85$ ) with $8.0 \pm 2.4$ years for nonfluent agrammatic variant PPA, 8.1 \pm 1.6 years for logopenic variant PPA, and $8.4 \pm 2.0$ years for semantic variant PPA. Informed consent was obtained from the patients or their spouses for those with severe cognitive impairment. Among the initial 914 patients with aphasia, 6 patients with behavior variant frontotemporal dementia later developed non-fluent agrammatic progressive aphasic symptoms and 5 patients with posterior cortical atrophy developed transcortical sensory aphasic symptoms as their diseases progressed. These 11 patients, however, did not developed logoclonia during the observation period of $8.3 \pm 3.0$ years. Patients with non-progressive aphasia were followed for approximately 2 years, who, contrary to those with progressive aphasia, improved their linguistic function over time. None of them presented with logoclonia during the observation period.

This report was written after obtaining informed consent from the patients.

\section{Methods}

We obtained a detailed description of the progression to logoclonia for each patient. We also investigated the disease etiology, demographics, and severity of dementia for these five patients with logopenic variant PPA. Severity of dementia was rated with the clinical dementia rating scale (CDR) [21] at the initial assessment and when they were 
diagnosed with logoclonia. Also, the time from the onset of logopenic variant PPA to the onset of logoclonia was calculated. Regarding detailed linguistic function, we focused on longitudinal linguistic changes, including word comprehension, word repetition, and naming abilities, which were assessed at the initial assessment and at or immediately before the onset of logoclonia using the Standard Language Test of Aphasia [22]. Characteristics of phonological errors during the course of the disease were categorized as substitution, sequence, omission, and addition.

\section{RESULTS}

\section{Case presentations}

\section{Case 1}

A right-handed woman with 12 years of education complained of word-finding difficulties at age 57. She visited a local clinic at age 60 , where she was not diagnosed as having dementia. Her linguistic dysfunction deteriorated, and she visited Edogawa Hospital at age 62. Her orientation, episodic memory, insight, judgment, and behavior were well preserved and she lived independently. Her performance IQ on WAIS-III was 91, indicating that she had a normal non-verbal intelligence. Her physical and neurological examination revealed no remarkable findings and no signs of parkinsonism. Regarding linguistic function, however, her speech was interrupted by word-finding difficulties, phonological paraphasias, and fragmentary syllables, despite having normal articulation, prosody, and grammar. Her repetition was limited to one word. Her forward digit span was a maximum of two digits. When asked to repeat "soraga aoi" (The sky is blue.), she said, "sorawo ... so ... so ... so", reflecting her remarkably impaired repetition. In naming tasks, she said "to" for "tokei" (watch), "chochii" for "chochin" (lantern), and, with an initial letter cue, "hikohshi, hikoh, hikohki" for "hikoki" (airplane). Phonological paraphasias and fragmentary syllables were found in all speech output modalities, i.e., naming, repetition, oral reading, and writing. Meanwhile, her comprehension of single words in both spoken and written form was intact, although comprehension of sentences for both language forms was sometimes impaired. Her writing abilities were also affected, with frequent phonological errors along with difficulties in remembering Chinese characters (The Japanese writing system uses a combination of both ideograms from Chinese characters, Kanji, and syllabic kana) because Chinese characters are more visually complex than syllabic kana. In addition to aphasia, she also had increased difficulties with arithmetic and manipulation of her personal computer. Her brain magnetic resonance imaging (MRI) showed atrophy mainly in the left temporo-parietal areas. $N$-isopropyl- $p$ - $\left[{ }^{123} \mathrm{I}\right]-$ iodoamphetamine single-photon emission computed tomography (SPECT) with three-dimensional stereotactic surface projection analysis at the age of 63 showed relative hypoperfusion, mainly in the left temporo-parietal junction (Fig. 1). She was aware of her aphasia and was frequently frustrated by her situ-

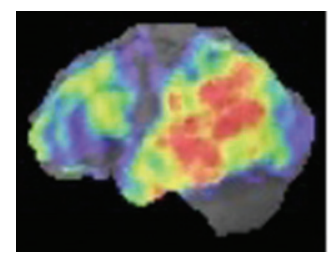

Left

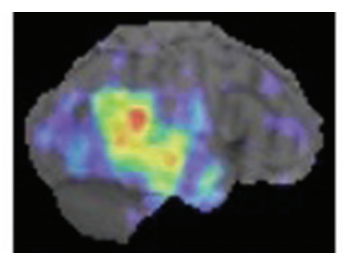

Right

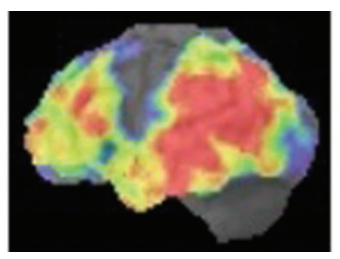

Left

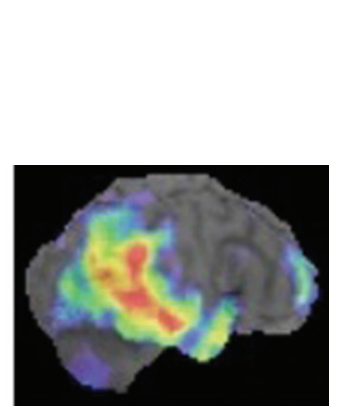

Right

Fig. 1. SPECT with $99 \mathrm{mTc}$-ethylcysteinate dimer for cases 2,3 , and 4 and with $N$-isopropyl-p-[ $\left.{ }^{123} \mathrm{I}\right]$-iodoamphetamine dimer for case 1 showed relative hypoperfusion mainly in the left temporo-parietal junction. The Z-score scale of 1 to 7 , which reflects lower regional cerebral blood flow, is indicated by the blue to red color gradient with a higher Z-score scale representing a lower reginal cerebral blood flow for case 1. For case 2 to 4 , the Z-score scale of 2 to 5 is indicated by the black to red color gradient. Case 1 . The left two scans were performed four year before her logoclonia appeared, whereas the right ones were performed when she developed logoclonia. 


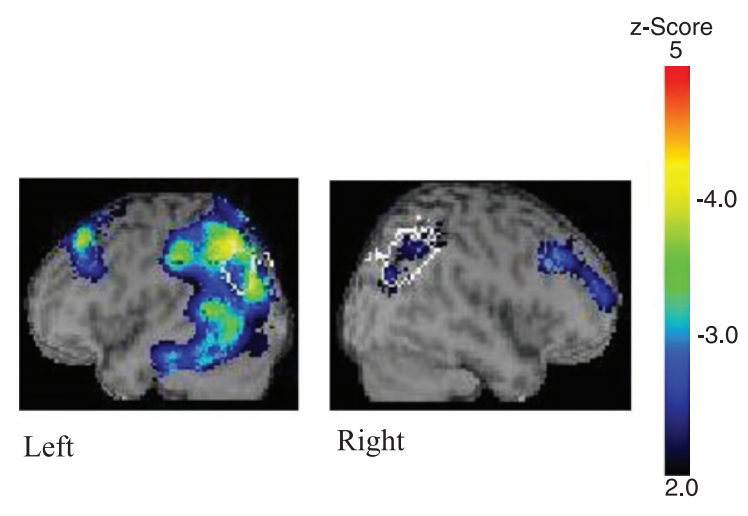

Fig. 2. Case 2. The two scans were performed one year before her logoclonia appeared.

ation. She began to undergo linguistic rehabilitation twice a week.

Although her aphasia temporally improved 6 months after commencing linguistic rehabilitation, it deteriorated again mainly with respect to the phonology-related domain, e.g., word repetition, word retrieval, and oral reading. She presented with mild episodic memory deficits at age 63. At age 64 , she could not retrieve most of the words in the naming task in the Standard Language Test of Aphasia and had occasional phonological paraphasia. At age 65 , she would get lost when she was alone outside, and she became unable to communicate with respect to her daily tasks. At age 67, she revealed conceptual apraxia, had difficulties in using tools due to impaired knowledge of tool action, and repeatedly used hand cream to clean utensils. At that time, she did not speak much and began to present with logoclonia during her daily conversations, such as "amatatotototototo" or "nakaratsutsutsutsutsutsuchachachachachacha". When logoclonia occurred, her speech would persist for extended periods. She required nursing care to complete most of her daily activities. She did not, however, present with frontal release signs, i.e., grasp reflex, instinctive grasp reflex, or utilization behavior. Her SPECT at the age of 67, when she presented with logoclonia, revealed that the degree of hypoperfusion in the left temporal and parietal areas had increased substantially.

\section{Case 2}

A 72-year-old right-handed woman with 12 years of education began to show word-finding difficulties with prolonged anomic pauses, which were partly compensated for by frequent pronouns. She also complained of difficulties in writing Chinese characters because of their complexity. She visited Edogawa Hospital at age 75. Her neurological examinations were normal, and she showed no signs of parkinsonism. When first assessed linguistically, she had a fluent aphasia with circumlocutory speech along with occasional lexical paraphasias. Upon assessment, she was able to successfully repeat single words, but her ability to repeat a sentence was impaired. Her forward digit span was a maximum of five digits. Comprehension of single words in both spoken and written form remained intact, whereas comprehension of complex sentences in both language forms was sometimes impaired. Her writing skills were also poor with phonological paragraphias and difficulties in remembering Chinese characters. Despite linguistic dysfunction, she was able to lead an independent life. There were no significant difficulties with episodic memory, and she had never become lost. She was aware of her condition. At the age of 75 , her brain MRI showed atrophy mainly in the left temporo-parietal areas. SPECT with $99 \mathrm{mTc}$ ethylcysteinate dimer with easy z-score imaging system (eZIS) showed relative hypoperfusion mainly in the left temporo-parietal junction (Fig. 2). She began linguistic rehabilitation once a week.

At age 76, her aphasia worsened and included circumlocution, verbal paraphasias, and phonological paraphasias. Phonological paraphasias were found in repetition, naming, and oral reading. Notably, she presented with phonological addition errors, e.g., "mimimizuku" for "mimizuku" (owl), along with phonological substitution errors, e.g., "taigyo" for "taiyo" (sun). At this time, she began to show episodic memory deficits and conceptual apraxia, i.e., a loss of knowledge of how objects are used, and she often got lost. At age 79, her speech consisted mainly of logoclonia, which persisted once she began, e.g., "Nakagawasesesese, seseseseh" (when she attempted to say "you are Dr. Nakagawa" or "Nakagawa-sensei" in Japanese) and "Atatakakaitachine, kototototo. Suhdededede, damenano" (which we were not able to understand). She was not able to live independently and needed nursing care to complete most of her daily activities. She did not, however, present with frontal release signs, i.e., grasp reflex, instinctive grasp reflex, or utilization behavior.

\section{Case 3}

A 59-year-old right-handed man with 16 years of education showed word-finding difficulties with prolonged anomic pauses. He had been a former office manager and was a community leader at that 


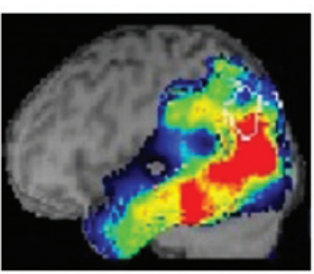

Left

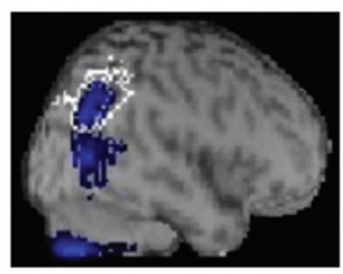

Right

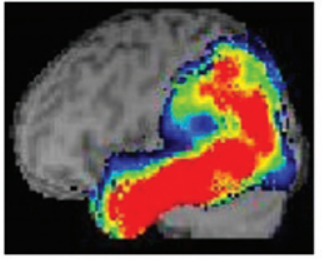

Left
z-Score

5

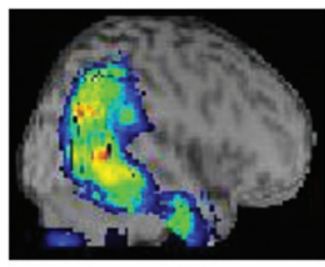

Right

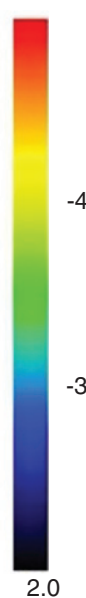

Fig. 3. Case 3. The left two scans were performed two year before his logoclonia appeared, whereas the right ones were performed when he developed logoclonia.

time, but he quit his role as a leader due to his progressive aphasia. He did, however, lead an independent life with no episodic memory deficits and was aware of his condition. At age 64, he was diagnosed as having logopenic variant PPA at a clinic that specializes in dementia in central Tokyo and that he had visited for 2 years. At age 66, he was referred to the cognitive function clinic associated with Ashikaga Red Cross Hospital to receive continuing linguistic rehabilitation in his hometown. When first assessed, his speech was fluent without apraxia of speech but was interspersed with circumlocutions, verbal paraphasias, and occasional phonological paraphasias. Phonological paraphasias were found mainly in naming but also occasionally in repetition and oral reading. Repetition was limited to three or four words, and his forward digit span was a maximum of four digits and backward digit span was only two digits. Although comprehension of single words was preserved, he sometimes had difficulties in understanding long sentences, which also applied to written language. Although he was aware of his anomia, he was relatively unaware of his comprehension difficulties. His brain MRI showed atrophy mainly in the left temporo-parietal areas. Although he showed mild episodic memory deficits, he lived independently. SPECT with 99mTc-ethylcysteinate dimer with eZIS analysis at the age of 64 showed relative hypoperfusion in the bilateral temporo-parietal areas (Fig. 3).

Over the years, his aphasia gradually worsened, and at age 66 he began to have difficulties using electronic appliances. His speech frequently involved phonological paraphasias, e.g., "daidoukoufu" for "daidokoro" (kitchen) and "habifura" for "haburashi" (toothbrush), and occasionally formal paraphasias, e.g., "apato" (apartment) for "depato" (department store), in which a word is replaced with another that is phonologically related to the intended word. Phonological paraphasias were found in all modalities, i.e., repetition, naming, oral reading, and writing. He sometimes got lost at age 67 and showed difficulties in how to dress, e.g., trying to wear a shirt over his pajamas. At age 68, he was sometimes unable to recognize even his family members. He also developed conceptual apraxia and he was unable to use almost all the tools of everyday life. He did not, however, present with frontal release signs, i.e., grasp reflex, instinctive grasp reflex, or utilization behavior. At that time, he occasionally presented with logoclonia, e.g., "shinbunbunbun" for "shinbun" (newspaper) and "fukesensen" for "fusen" (balloon), which were sometimes undistinguishable from phonological addition errors. SPECT with $99 \mathrm{mTc}$-ethylcysteinate dimer with eZIS analysis at the age of 68 , when he presented with logoclonia, revealed that the areas of hypoperfusion in the left temporo-parietal areas had increased substantially to the point where the whole left temporal and inferior parietal areas were severely affected.

\section{Case 4}

A 59-year-old right-handed woman with 12 years of education complained of having difficulties with 


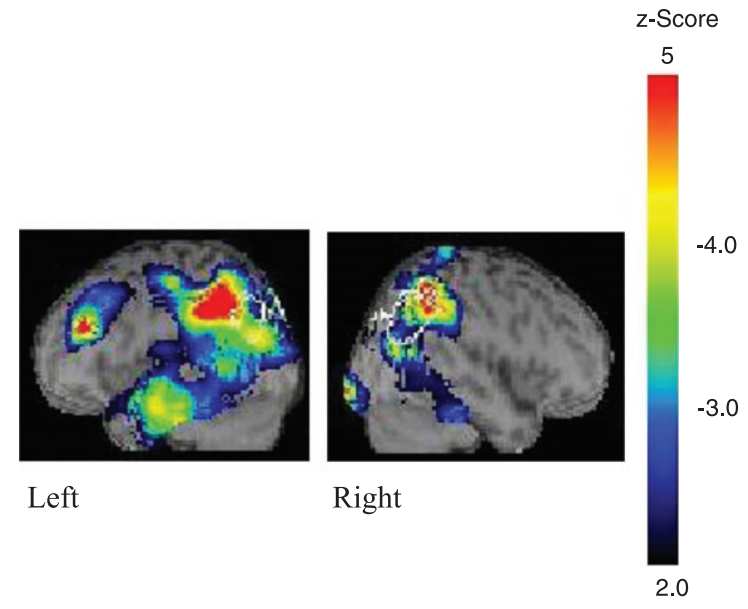

Fig. 4. Case 4. The two scans were performed one year before her logoclonia appeared.

word finding and manipulating electronic appliances. Her husband noticed that although she fully comprehended daily conversation, she had difficulties in remembering proper nouns, e.g., her friends' names. At age 61, she was diagnosed as having mild cognitive impairment at a local clinic. Her linguistic abilities deteriorated despite her episodic memory function remaining intact. Thus, her husband asked for a more specialized assessment, and she was referred to the cognitive function clinic associated with Ashikaga Red Cross Hospital at age 63. At initial assessment, she had a fluent aphasia with circumlocutory speech along with occasional phonological paraphasias, such as "tomai" for "tomodachi" (friend). Repetition of single words was preserved but sentence repetition was limited to three words. Comprehension of single words in both spoken and written form remained intact, whereas comprehension of complex sentences in either form was sometimes impaired. Although she was aware of her aphasia for the most part, she declined to perform further linguistic assessment. Her brain MRI showed atrophy mainly in the left temporo-parietal areas. SPECT with 99mTcethylcysteinate dimer with eZIS analysis at the age of 64 showed relative hypoperfusion in the bilateral temporo-parietal areas (Fig. 4). She showed mild episodic memory deficits and she forgot how to cook familiar dishes. She required some assistance to complete daily activities.

Over the course of the disease, her aphasia gradually worsened, and she was unable to repeat two words at age 64. She sometimes had difficulties in comprehending even single words. Her speech was interspersed with many circumlocutions and phonological paraphasias, which were mainly substitution errors of speech sounds. Her vocabulary became extremely limited, with only basic conversational skills. She sometimes got lost and showed episodic memory deficits and conceptual apraxia. At age 65, her speech consisted of logoclonia, e.g., "matetetetetetete" for "mate" (wait), "memeganenenenenenenetetetete" for "megane" (a pair of glasses), and "urusaisaisaisaisaisaisaisai!" for "urusai" (Be quiet!). She rarely spoke, but once she started speaking, her speech was persistent. At this time, she required complete care for her daily activities. She did not, however, present with frontal release signs, i.e., grasp reflex, instinctive grasp reflex, or utilization behavior.

\section{Case 5}

This case report has already been described elsewhere [23]. In short, a right-handed man with logopenic variant PPA noticed progressive speech difficulties at age 51 and progressed into neologistic jargon with remarkable phonological addition errors at age 55. He was aware of and often irritated by his aphasia. At age 58, he did not speak much, and most of his speech consisted of logoclonia, such as "sonnnanananananana" and "sirorourorororo". Based on our clinical observations, phonological addition errors most likely developed into logoclonia. He was no longer able to comprehend many single words. At age 59, he gradually became confused with what to use for a desired action. For example, he confused toothpaste with shaving cream.

\section{Case summary and linguistic function assessed with the Standard Language Test of Aphasia}

Tables 1 and 2 show the linguistic function at the initial stages and at the onset of logoclonia, respectively, for all five individuals described here. All five patients with logoclonia were diagnosed with logopenic variant PPA at the initial stages and met the clinical criteria for a diagnosis of probable Alzheimer's disease; in particular, four of the five patients had early-onset Alzheimer's disease (Table 1). During the course of the disease, phonological function deteriorated with various types of phonological errors, which were found in all modalities, i.e., repetition, naming, and oral reading (Table 1). Among several types of phonological paraphasias, substitution and addition were frequently found in these patients. In particular, phonological 
Table 1

Demographics and linguistic function as assessed by the SLTA at initial assessment

\begin{tabular}{|c|c|c|c|c|c|}
\hline Characteristic & Case 1 & Case 2 & Case 3 & Case 4 & Case 5 \\
\hline Aphasia type & lvPPA & lvPPA & lvPPA & lvPPA & lvPPA \\
\hline Age of onset, y/gender & $57 / \mathrm{F}$ & $72 / \mathrm{F}$ & $59 / \mathrm{M}$ & $59 / \mathrm{F}$ & $51 / \mathrm{M}$ \\
\hline Education, y & 12 & 16 & 16 & 12 & 12 \\
\hline Clinical diagnosis & Early-onset $\mathrm{AD}$ & Late-onset AD & Early-onset AD & Early-onset $\mathrm{AD}$ & Early-onset $\mathrm{AD}$ \\
\hline $\operatorname{CDR}(0.5$, very mild to 3 , severe $)$ & 0.5 & 0.5 & 0.5 & 0.5 & 0.5 \\
\hline Forward digit span & 2 & 5 & 4 & NA & 3 \\
\hline Word comprehension, \% correct & 100 & 100 & 100 & NA & 100 \\
\hline Word repetition, \% correct & 90 & 100 & 100 & NA & 80 \\
\hline Confrontation naming, $\%$ correct & 65 & 65 & 45 & NA & 35 \\
\hline $\begin{array}{l}\text { Type of phonological } \\
\text { paraphasias* }\end{array}$ & $\begin{array}{l}\text { Substitution, omission, } \\
\text { fragmentary syllables }\end{array}$ & $\begin{array}{l}\text { Substitution, } \\
\text { addition }\end{array}$ & $\begin{array}{l}\text { Substitution, } \\
\text { addition }\end{array}$ & $\begin{array}{l}\text { Substitution, } \\
\text { addition }\end{array}$ & $\begin{array}{l}\text { Substitution, } \\
\text { addition }\end{array}$ \\
\hline
\end{tabular}

*The type of phonological paraphasia was determined during both the initial assessment and a subsequent assessment. lvPPA, logopenic variant primary progressive aphasia; CDR, Clinical Dementia Rating scale; SLTA, Standard Language Test of Aphasia in Japanese; AD, Alzheimer's disease; NA, not assessed.

Table 2

Linguistic function as assessed by the SLTA at onset of logoclonia

\begin{tabular}{|c|c|c|c|c|c|}
\hline Linguistic characteristic & Case 1 & Case 2 & Case 3 & Case 4 & Case 5 \\
\hline Onset of logoclonia & 10 years later & 7 years later & 9 years later & 6 years later & 7 years later \\
\hline Word comprehension, $\%$ correct & 70 & 70 & 100 & NA & 90 \\
\hline Word repetition, \% correct & 20 & 100 & 90 & NA & 60 \\
\hline Confrontation naming, $\%$ correct & 0 & 30 & 0 & NA & 0 \\
\hline Apraxia of speech & Not found & Not found & Not found & Not found & Not found \\
\hline CDR $(0.5$, mild to 3 , severe $)$ & 3 & 3 & 3 & 3 & 3 \\
\hline Jargon & Logoclonia & $\begin{array}{c}\text { Logoclonia and } \\
\text { undifferentiated jargon }\end{array}$ & Logoclonia & Logoclonia & $\begin{array}{c}\text { Logoclonia and } \\
\text { neologistic jargon }\end{array}$ \\
\hline
\end{tabular}

CDR, Clinical Dementia Rating scale; SLTA, Standard Language Test for Aphasia in Japanese; NA, not assessed.

addition errors were closely related with logoclonia in Cases 3 and 5. All five individuals developed logoclonia an average of 7.8 years post-onset, when their cognition was severely impaired, i.e., at a score of 3 on the Clinical Dementia Rating scale (Table 2). They also developed conceptual apraxia, i.e., a loss of knowledge of how objects are used. During the course of progressing from logopenic variant PPA to logoclonia, despite not having apraxia of speech, their naming abilities became extremely poor with almost no correct answers when compared with their word comprehension and word repetition abilities (Table 2), suggesting their severe phonological output dysfunction. Although the five patients did not speak much at the advanced stage, three of the five patients showed voluminous speech with logoclonia once they started speaking.

SPECT images of four of the patients showed left temporo-parietal hypoperfusion, a characteristic imaging finding for patients with logopenic variant PPA, and the longitudinal SPECT scans obtained from cases 1 and 3 (Figs. 1, 3) showed that hypoperfusion in the left temporal and inferior parietal areas had deteriorated substantially, suggesting that the linguistic functions, such as naming abilities [24-26] and phonological function [26-29], might be severely affected, whereas articulation and syntax, which are closely related with Broca's area [30], might be preserved (Fig. 1).

\section{DISCUSSION}

Two findings arise from our results. First, logoclonia was found only in the advanced stages of logopenic variant PPA, and its etiology was most likely Alzheimer's disease, which is consistent with previous research [2-6]. Second, the development of logoclonia was associated with extremely reduced naming abilities and phonological dysfunction, which can be related to dysfunction in the left temporal and left inferior parietal areas.

The fact that logoclonia was found only in individuals with logopenic variant PPA versus non-fluent agrammatic or semantic variants suggests potential mechanisms underlying logoclonia. In the present cases, comprehension of individual words was relatively preserved when compared with extremely reduced naming abilities, suggesting that storage of lexical information might be retained to some extent 
in our patients when compared with the semantic variant PPA, which is characterized by reduced storage of lexical and semantic information [31]. Rather, deficits in phonology are considered a hallmark of logopenic variant PPA [19]. The phonological dysfunction might give us a clue to possible mechanisms behind logoclonia. In the present patients, the repetition of single words was preserved, in contrast to their extremely poor naming abilities. This might reflect deficits in the phonological production process. There are different mechanisms for naming and word repetition $[32,33]$. The process of picture naming is often viewed as involving both a semantic and phonological step, i.e., a two-step process [32]. Word repetition, in contrast, may be accomplished by direct mapping of input phonology (the sounds that make up the heard word) to output phonology, without the involvement of either of the two naming steps [33]. Alternatively, it could incorporate the phonological step, such that the heard word is first mapped onto its abstract representation, and then its output phonology is retrieved via the phonological step [33]. For either hypothesis, as compared with repetition, naming requires more extensive processing, i.e., the semantic step and the phonological production process. The fact that the phonological paraphasias of these five patients were found not only during repetition but also during naming, oral reading, and even writing suggests that their deficits involve the phonological production process [27-29] as well as phonological short-term memory, which is represented by repetition. This phonological production process is considered to involve the Wernicke's area and the left inferior parietal area [26-29], that is, the left temporo-parietal junction, which was shown to be severely affected in the brain scans obtained from our patients (Fig. 1). Dysfunction of the semantic step during naming can be represented as word retrieval deficits, which involves word-finding difficulties and verbal paraphasias without any phonological paraphasias. Such deficits have been associated with the left middle temporal gyrus, an area anteroinferior to the left temporo-parietal junction [24-26]. Hypoperfusion in this area and even deterioration of the hypoperfusion after the two patients developed logoclonia were also found in our patients' brain scans, which suggested that they had dysfunction in the semantic step in naming as well as phonological output dysfunction. Involvement of conceptual apraxia in these patients is compatible with the impaired semantics.

Although they did not speak much at the advanced stage, three patients showed voluminous speech with logoclonia once they did start speaking. Voluminous speech has been noted in jargon aphasia [34], which was explained by the authors as resulting from an absent "brake" to stop speech generation. A similar mechanism might have led to the voluminous speech in our patients. In fact, Broca's area in the left inferior frontal gyrus in these patients was relatively intact when compared with the temporal and parietal regions (Fig. 1). This neuroanatomical finding is compatible with the fluent and sporadic voluminous speech in our patients.

What would happen if deficits of the semantic step in naming and phonological production process gradually deteriorated as occurred in these five patients? Speed and/or the variety of phonological production might be slowed or lost, which could result in a paucity of phoneme[s] as the name "logopenic" implies. In this situation, a slow and narrow range of phoneme choice along with intact fluency (no apraxia of speech) and/or the absence of a brake to stop speech might lead to continuity of the same phoneme or same sounds in a word, i.e., logoclonia. Although logoclonia has been associated with perseveration, deterioration in phonological output function and naming abilities along with retained speech fluency might be the basis for the development of logoclonia. A close relationship between phonological addition errors and logoclonia in some patients might also support this explanation, because phonological addition errors are regarded as phonological impairment but not as perseveration [35-37].

Our study has several limitations that should be considered. First, the number of patients with logoclonia is small, which reflects the rareness of this condition, with only 5 of 914 aphasic patients showing this sign. Second, the reason why logoclonia occurred only in patients with degenerative diseases was unclear. Logoclonia did not occur in individuals with vascular aphasias, i.e., conduction aphasia or Wernicke's aphasia, conditions that also have phonological output deficits. This might reflect differences in the nature of these diseases, in degeneration versus vascular diseases, or in diffuse atrophy versus focal damage. Third, only 5 of 16 patients with logopenic variant PPA presented with logoclonia. This might be attributable to heterogeneity of logopenic variant PPA [38]. Another possibility might be that the observation period was not long enough, because those patients who did develop logoclonia showed signs an average of 7.8 years post-onset and 5 of 16 patients had not been followed up for more than 7 years. Lastly, our study did not include non-aphasic 
degenerative patients, who might also present with logoclonia, in particular, degenerative extrapyramidal syndrome.

\section{Conclusions}

Despite these limitations, our study found that logoclonia might be a characteristic sign of logopenic variant PPA at advanced stages. Although logoclonia might be associated with perseveration, deterioration in naming abilities and phonological output function along with retained speech fluency might lead to its development.

\section{ACKNOWLEDGMENTS}

We thank the patients and their family members for allowing us to report the results of their case studies. The authors have no funding to declare.

Authors' disclosures available online (https:// www.j-alz.com/manuscript-disclosures/19-0184r2).

\section{REFERENCES}

[1] Kraepelin E (1910) Psychiatrie, 8 te Aufl., Johann Ambrosius Barth, Leipzig.

[2] Foix C, Chavany JA (1926) Palilalie syllabique. Selerose intracérébrale en foyers disséminés. Rev Neurol 33, 61-68.

[3] Gustafson L, Nilsson L (1982) Differential diagnosis of presenile dementia on clinical grounds. Acta Psychiatr Scand, 65, 194-209.

[4] Gustafson L, Brun A, Endlund E, Hagnell O, Nilsson K, Stensmyr M, Ohlin AK, Abrahamson M (1998) A 50year perspective of a family with chromosome-14-kinked Alzheimer's disease. Hum Genet 102, 253-257.

[5] Amano N, Yagishita S (2005) A 74-year-old woman presenting slowly progressive dementia and emotional instability. Neuropathology 25, 107-109.

[6] Appel J, Kertesz A, Fismen M (1982) A study of language functioning in Alzheimer patients. Brain Lang 17, 73-91.

[7] Wallesch CW, Papagno C, Blanken G (1990) Dissociations of language functions in aphasics with speech automatisms (recurring utterances). Cortex 26, 41-63.

[8] Wallesch CW, Blanken G (2000) Recurring utterances-how, where, and why are they generated? Brain Lang 71, 255257.

[9] Kubo S, Hattori T, Takanashi M, Mori H, Mizutani Y, Suda K, Hattori N, Mizuno Y (2000) A 57-year-old woman with progressive disturbance of gait and mental deterioration. Brain Nerve 52, 1035-1044.

[10] Critchle M (1947) Post-encephalitic parkinsonism with marked palilalia. Proc R Soc Med 40, 552.

[11] Koller WC (1983) Dysfluency (stuttering) in extrapyramidal disease. Arch Neurol 40, 175-177.

[12] Uyama E, Katahira T, Okada H, Hashimoto Y, Araki S (1989) Palilalia associated with progressive supranuclear palsy (Japanese). Rinshi Shinkeigaku 29, 617-621.
[13] Sakai T, Miyamura M, Kuzuhara S (1992) Palilalia and acquired stuttering in a case of Parkinson's disease (Japanese). Rinsho Shinkeigaku 32, 859-863.

[14] Leder SB (1996) Adult onset of stuttering as a presenting sign in a parkinsonian-like syndrome: A case report. J Commun Disord 29, 471-477.

[15] Anderson JM, Hughes JD, Gonzalez Rothi LJ, Crcian GP, Heilman KM (1999) Developmental stuttering and Parkinson's disease: The effects of levodopa treatment. J Neurol Neurosurg Psychiatry 66, 776-778.

[16] Kikuchi Y, Umezaki T, Uehara T, Yamaguchi H, Yamashita K, Hiwatashi A, Sawatsubashi M, Adachi K, Yamaguchi Y, Murakami D, Kira JI, Nakagawa T (2018) A case of multiple system atrophy-parkinsonian type with stuttering- and palilalia-like dysfluencies and putaminal atrophy. J Fluency Discord 57, 2018.

[17] Joseph R (1999) Frontal lobe psychopathology: Mania, depression, confabulation, catatonia, perseveration, obsessive compulsions, and schizophrenia. Psychiatry 62, 138-172.

[18] Ioannidis P, Konstantinopoulou E, Karacostas D (2015) Drawing perseveration in the behavioral variant of frontotemporal dementia. Neurology 84, 101.

[19] Gorno-Tempini ML, Hillis AE, Weintraub S, Kertesz A, Mendez M, Cappa SF, Ogar JM, Rohrer JD, Black S, Boeve BF, Manes F, Dronkers NF, Vandenberghe R, Rascovsky K, Patterson K, Miller BL, Knopman DS, Hodges JR, Mesulam MM, Grossman M (2011) Classification of primary progressive aphasia and its variants. Neurology 76, 1006-1014.

[20] Gorno-Tempini ML, Brambati SM, Ginex V, Ogar J, Dronkers NF, Marcone A, Perani D, Garibotto V, Cappa SF, Miller BL (2008) The logopenic/phonological variant of primary progressive aphasia. Neurology 71, 1227-1234.

[21] Morris JC (1993) The Clinical Dementia Rating (CDR): Current version and scoring rules. Neurology 43, 24122414.

[22] Hasegawa T, Kishi H, Shigeno K, Tanemura J, Kusunoki $\mathrm{T}$ (1985) Three-dimensional structure in language tests of aphasia. Folia Phoniatr (Basel) 37, 246-258.

[23] Funayama M, Nakagawa Y, Yamaya Y, Yoshino F, Mimura M, Kato M (2013) Progression of logopenic variant primary progressive aphasia to apraxia and semantic memory deficits. BMC Neurol 13, 158.

[24] Schwartz MF, Kimberg DY, Walker GM, Faseyitan O, Brecher A, Dell GS, Coslett HB (2009) Anterior temporal involvement in semantic word retrieval: Voxel-based lesion-symptom mapping evidence from aphasia. Brain 132, 3411-3427.

[25] Baldo JV, Arévalo A, Patterson JP, Dronkers NF (2013) Grey and white matter correlates of picture naming: Evidence from a voxel-based lesion analysis of the Boston Naming Test. Cortex 49, 658-667.

[26] Butler RA, Lambon Ralph MA, Woollams AM (2014) Capturing multidimensionality in stroke aphasia: Mapping principal behavioural components to neural structures. Brain 137, 3248-3266

[27] Shallice T, Rumiati RI, Zadini A (2000) The selective impairment of the phonological output buffer. Cogn Neuropsychol 17, 517-546.

[28] Binder JR (2017) Current controversies on Wernicke's area and its role in language. Curr Neurol Neurosci Rep 17, 58.

[29] Fridriksson J, Basilakos A, Hickok G, Bonilha L, Rorden C (2015) Speech entrainment compensates for Broca's area damage. Cortex 69, 68-75. 
[30] Balasubramanian V (2005) Dysgraphia in two forms of conduction aphasia. Brain Cogn 57, 8-15.

[31] Landin-Romero R, Tan R, Hodges JR, Kumfor F (2016) An update on semantic dementia: Genetics, imaging, and pathology. Alzheimers Res Ther 8, 52.

[32] Schriefers H, Meyer AS, Levelt WJM (1990) Exploring the time course of lexical access in language production: Picture-word interference studies. J Mem Lang 29, 1990.

[33] Nozari N, Kittredge AK, Dell GS, Schwartz MF (2010) Naming and repetition in aphasia: Steps, routes, and frequency effects. J Mem Lang 63, 541-559.

[34] Robinson GA, Butterworth B, Cipolotti L (2015) "My mind is doing it all": No "brake" to stop speech generation in jargon aphasia. Cogn Behav Neurol 28, 229-241.
[35] Ardila A, Montanes P, Caro C, Delgado R, Buckingham HW (1989) Phonological transformations in Spanish-speaking aphasics. J Psycholinguist Res 18, 163-180.

[36] Santos FH, Bueno OF, Gathercole SE (2006) Errors in nonword repetition: Bridging short- and long-term memory. Braz J Med Biol Res 39, 371-84.

[37] Dalton SGH, Shultz C, Henry ML, Hillis AE, Richardson JD (2018) Describing phonological paraphasias in three variants of primary progressive aphasia. Am J Speech Lang Pathol 27, 336-346.

[38] Leyton EC, Hodges JR, McLean CA, Kril JJ, Piguet O, Ballard KJ (2015) In the logopenic-variant of primary progressive aphasia a unitary disorder? Cortex 67, 122-133. 